\title{
Lattice Gauge Theory and Two Dimensional Quantum Gravity
}

\author{
Simon Catterall*, Judah Unmuth-Yockey and Muhammad Asaduzzaman \\ Department of Physics, Syracuse University, Syracuse, New York 13244, United States \\ E-mail: smcatteresyr.edu, jfunmuthesyr.edu, masaduzzesyr.edu
}

\begin{abstract}
We construct a two dimensional $S U(2)$ lattice gauge theory containing a single adjoint scalar field which, when tuned to a critical point associated to spontaneous breaking of center symmetry, possesses a long distance effective theory which describes two dimensional quantum gravity. The metric is emergent in the theory and depends on the gauge fields with the diffeomorphism symmetry of the continuum metric theory arising from lattice gauge symmetry. Apart from the intrinsic interest in formulating gravity as a lattice gauge theory the construction holds some advantages over traditional numerical approaches eg. dynamical triangulations since it uses only conventional cubic lattices and Wilson gauge links.
\end{abstract}

37th International Symposium on Lattice Field Theory - Lattice2019

16-22 June 2019

Wuhan, China

${ }^{*}$ Speaker. 


\section{Introduction and Motiovation}

Two dimensional quantum gravity is a useful toy model for understanding certain aspects of gravity in four dimensions. The pure gravity theory is both exactly soluble and non-trivial. It can also be coupled to conformal matter at least for central charge less than one.

The central goal is to compute the Feynman path integral corresponding to a sum over two dimensional metrics modulo diffeomorphisms. This program can be carried out in the continuum and results in a effective theory for gravity in which the conformal factor of the metric acquires non-trivial dynamics determined by the Liouville/Polyakov action $[1,2,3]$. Upon integration over the conformal factor a non-local, coordinate invariant action is produced.

This path integral can also be computed using a matrix integral in the large $N$ limit with the Feynman graphs occurring in the perturbative expansion of the matrix model approximating discrete two dimensional geometries [4]. The duals to such graphs realize random triangulations and can be sampled using Monte Carlo simulation [6, 5, 7].

In this paper we will show that two dimensional quantum gravity can also arise at a critical point of a particular lattice gauge theory. We start by rewriting Einstein gravity in the Cartan formalism using a frame and spin connection. We then follow MacDowell, Chamseddine and others $[8,9]$ and show that this theory can be realized as a Higgs phase of $S U(2)$ gauge theory containing an additional scalar field.

We then use the usual prescription to discretize this theory on a lattice. The resultant theory is topological as expected for all two dimensional gauge theories. However there is a new marginal operator that can be added to the lattice theory that while breaking coordinate invariance respects all the lattice symmetries. We then construct an effective theory by integrating out the scalar field and show in a particular scaling limit the effective gauge action is non other than the Polyakov action of two dimensional gravity. Furthermore the unique coupling characterizing the gravity theory which is determined by the conformal anomaly is obtained by tuning the lattice theory to a phase transition at which spontaneous breaking of a $Z_{2}$ center symmetry occurs.

\section{Cartan gravity in two dimensions}

As a first step we introduce the frame form $e_{\mu}^{a}$ in terms of the metric $g_{\mu \nu}$ by

$$
g_{\mu v}(x)=\sum_{a=1}^{2} e_{\mu}^{a}(x) e_{v}^{a}(x)
$$

Clearly this decomposition is invariant under the local (Euclidean) $S O(2)$ Lorentz transformation

$$
e_{\mu}^{a}(x) \rightarrow \Lambda^{a b}(x) e_{\mu}^{b}(x)
$$

To account for this gauge invariance we must introduce a corresponding gauge field called the spin connection $\omega_{\mu}=\sum_{a<b} \omega_{\mu}^{a b} T^{a b}$ with $T^{12}=\sigma_{3}$ the generator of the $S O(2)$ group. The corresponding field strength is $R_{\mu v}=\left[D_{\mu}, D_{v}\right]$ with the covariant derivative defined in the usual way: $D_{\mu}=$ $\partial_{\mu}+\omega_{\mu}$. From these ingredients the simplest coordinate invariant action we can form in two dimensions is

$$
S=\int d^{2} x \varepsilon^{\mu v} \varepsilon_{a b}\left(R_{\mu v}^{a b}+\frac{1}{\ell^{2}} e_{[\mu} e_{v]}\right)
$$


Treating the frame $e_{\mu}^{a}$ as a $2 \times 2$ matrix the second term in this expression is nothing more than $2 \operatorname{det}(e)=2 \sqrt{\operatorname{det}(g)}$ and this expression clearly corresponds to a cosmological constant term with $\frac{2}{\ell^{2}}$ playing the role of the cosmological constant. Similarly the first term can be recognized as $2 \int \sqrt{\operatorname{det}(\mathrm{g})} R$ once one employs the identity

$$
\varepsilon^{\mu v}=\varepsilon^{a^{\prime} b^{\prime}} e_{a^{\prime}}^{\mu} e_{b^{\prime}}^{v} \operatorname{det}(\mathrm{e})
$$

In the last expression the notation $e_{a}^{\mu}$ denotes the inverse of the $2 \times 2$ matrix $e_{\mu}^{a}$. These manipulations suggest that the two formalisms are identical as long as the frame can be inverted. Actually this is not quite true; the field strength appearing in eqn. 2.3 is a function of the spin connection not the metric. To complete the equivalence one needs to impose an additional condition that determines the spin connection as a function of the frame. This is the zero torsion condition $D_{[\mu} e_{v]}=0$.

Thus far the presentation is textbook and the correspondence between Cartan and Einstein gravity is well known. The Cartan formalism has an advantage over the Einstein theory in that it does not require the introduction of a background metric to contract indices - instead it resembles a topological theory requiring only the $\varepsilon$ symbol to construct a generally covariant theory. However, while the Cartan theory has the structure of a gauge theory, it requires both a gauge field and an additional vector field for its definition and in this way differs from a pure Yang-Mills theory. However, many years ago it was shown that Einstein-Hilbert theory could be obtained from a simpler Yang-Mills theory with an enhanced gauge symmetry - namely a theory where the Lorentz symmetry is extended to a de Sitter symmetry $[8,9]^{1}$. In this de Sitter gauge theory the gauge fields contain both the spin connection and the frame. In two (Euclidean) dimensions the required action is

$$
S=\int d^{2} x \varepsilon_{a b c} \varepsilon^{\mu v} F_{\mu \nu}^{a b} \phi^{c}
$$

here $F$ is the field strength corresponding to the $S O(3)$ de Sitter symmetry and the theory requires a additional scalar field $\phi$ living in the fundamental representation of the group. If we adopt a unitary gauge $\phi^{a}=\rho \delta^{3 a}$ the action collapses to

$$
S=\int d^{2} x \varepsilon_{a b} \varepsilon^{\mu v} \rho(x)\left(R_{\mu v}^{a b}-A_{[\mu} A_{v]}\right)
$$

If we then assume a spontaneous breaking $S O(3) \rightarrow S O(2)$ corresponding to $\rho(x)=1$ this action is none other than the $\mathrm{EH}$ action if we identify $e_{\mu}=\ell A_{\mu}$. Notice that the classical equations of motion following from eqn. 2.5 require all components of the field strength to vanish which implies in unitary gauge that both the Einstein equation and the torsion free condition hold:

$$
\begin{aligned}
R_{\mu v}^{a b}-\frac{1}{\ell^{2}} e_{[\mu}^{a} e_{v]}^{b} & =0 \\
D_{[\mu} e_{v]}^{a} & =0
\end{aligned}
$$

In practice it is possible to exploit the homomorphism $S O(3) \sim S U(2)$ and replace this with the simpler action

$$
S=\int d^{2} x \varepsilon^{\mu v} \operatorname{Tr}(F \phi)
$$

\footnotetext{
${ }^{1}$ In three dimensions Witten has shown that a similar construction realizes 3d gravity as a Chern-Simons theory [10]
} 
where $\phi$ is taken in the adjoint of the group. We will use this representation in what follows.

Of course $\int \sqrt{\operatorname{det}(\mathrm{g})} R$ is a topological invariant in two dimensions and so the classical gravity action for geometries of fixed topology and volume is trivial in two dimensions. However, as originally pointed out by Polyakov there is another marginal operator that can be considered

$$
\int d^{2} x \sqrt{g} \int d^{2} x^{\prime} \sqrt{g^{\prime}} R(x) \square^{-1}\left(x-x^{\prime}\right) R\left(x^{\prime}\right)
$$

Such an operator is naturally induced when massless matter fields are integrated out in a curved background. It also is needed to reproduce the effects of the two dimensional trace anomaly [11, 12]. The question we will address in the remainder of this paper is whether such a term can be generated within the context of the $S U(2)$ gauge theory formulation and specifically within a lattice realization of that theory.

\section{Lattice and extended gravity action}

In the original Cartan theory the choice of background space was arbitrary since only the epsilon symbol was needed to contract world indices. To make life as simple as possible we will now pick a flat background space. Given that, it is trivial to discretize the action given in eqn. 2.9 using a square lattice and employing a standard Wilson prescription for the gauge fields:

$$
S_{L}=\frac{1}{2} \sum_{x} \varepsilon^{\mu v} \operatorname{Re} \operatorname{Tr}\left(i U_{\mu v}^{P}(x) \phi(x)\right)
$$

where $U_{\mu \nu}^{P}(x)=U_{\mu}(x) U_{v}(x+\hat{\mu}) U_{\mu}^{\dagger}(x+\hat{v}) U_{v}^{\dagger}(x)$ is the usual Wilson plaquette formed by taking the product of $S U(2)$ group elements around an elementary square in the lattice.

Of course by going to a lattice we have broken the coordinate invariance of the theory. So we should ask ourselves what other counter terms should be included that are consistent with the exact lattice symmetries. Two come to mind; the usual Wilson plaquette term $\beta \sum_{x ; \mu \nu} \operatorname{Re} \operatorname{Tr}\left(U_{\mu \nu}^{P}\right)$ and a kinetic operator for the scalar field $\gamma \sum_{x} \operatorname{Tr}\left(D_{\mu} \phi\right)^{2}$. The former operator is irrelevant in two dimensions and hence should have no effect at long distances. However in the UV it plays an important role in keeping the fluctuations of the gauge field small as $\beta \rightarrow \infty$. This allows us to define a naive continuum limit in which our lattice expressions approach their continuum counterparts. The second term is a marginal operator ${ }^{2}$ that may be important in the long distance effective theory. The covariant difference operator appearing in this kinetic term is given by

$$
D_{\mu} \phi(x)=U_{\mu}(x) \phi(x+\hat{\mu}) U_{\mu}^{\dagger}(x)-\phi(x)
$$

It is instructive to examine this lattice action in unitary gauge which is gotten by setting

$$
\phi(x)=\frac{1}{\sqrt{2}} \rho(x) G(x) \sigma_{3} G(x)^{\dagger}
$$

where $G$ is an $S U(2)$ matrix and $\rho$ is a radial (Higgs-like) field. Inserting this expression into the lattice action, cyclically permuting the trace and changing variables to $U_{\mu} \rightarrow G^{\dagger} U_{\mu} G$ one finds:

$$
S=\sum_{x} \frac{1}{\sqrt{2}} \rho(x) \operatorname{Re} \operatorname{Tr}\left(i \sigma_{3} U_{12}(x)\right)-\frac{\beta}{2} \operatorname{Re} \operatorname{Tr}\left(U_{12}(x)\right)+\gamma \sum_{x} \rho(x)\left(-\square_{c}\right) \rho(x)
$$

\footnotetext{
${ }^{2}$ Notice that $\phi$ must be a dimensionless field for the correspondence to the EH theory to remain true.
} 
where the covariant difference operator applied to the field $\rho$ is given by

$$
\square_{c} \rho(x)=\sum_{\mu} V_{\mu}(x) \rho(x+\mu)+V_{\mu}(x-\mu) \rho(x-\mu)-2 \rho(x)
$$

and

$$
V_{\mu}(x)=\frac{1}{2} \operatorname{Tr}\left(\sigma_{3} U_{\mu}(x) \sigma_{3} U_{\mu}^{\dagger}(x)\right)
$$

with $U_{\mu}$ the gauge transformed link field. Setting $U_{\mu}(x)=e^{i \sum_{i=1}^{3} A_{\mu}^{i}(x) \sigma^{i}}$ we find for small $A_{\mu}^{i}(\beta \rightarrow$ $\infty)$

$$
V_{\mu}(x)=1-\left(A_{\mu}^{a}\right)^{2}+\ldots
$$

where the index $i \equiv a$ now runs only over the first two components of the field since $A_{\mu}^{3}$ remains massless in this gauge. Thus in unitary gauge the naive continuum limit of this new action reads

$$
S=\int d^{2} x\left[\sqrt{2} \rho(x) F_{12}^{3}(x)+\frac{1}{g^{2}} \operatorname{Tr}\left(F_{12}(x) F_{12}(x)\right)+\gamma \rho(x)\left(-\bar{\square}+m^{2}(A)\right) \rho(x)\right]
$$

with $\bar{\square}$ the usual free Laplacian in flat space, $m^{2}=2 \sum_{\mu}\left(A_{\mu}^{a}\right)^{2}$ and $g^{2}=\frac{1}{\beta}$ the dimensionful gauge coupling in two dimensions.

\section{The scaling limit}

To build the connection to gravity it is first necessary to rescale the pieces of the gauge field which will play the role of the frame $e_{\mu}$. These are precisely the components of the gauge field that appear in the action of the covariant Laplacian on the Higgs field in eqn. 3.8. Using this as motivation we will take $\gamma=b^{2}$ where $b=g \ell$ where $\ell$ is the linear size of the system. This will allow us to rewrite the gauge fields appearing in eqn. 3.8 as frame fields. Furthermore, we expect that this dimensionless parameter $b$ will govern the dynamics of the two dimensional theory. In the naive continuum limit the kinetic term in unitary gauge now reads

$$
\int d^{2} x\left(b^{2} \rho(-\bar{\square}) \rho+g^{2} \sum_{\mu} e_{\mu}^{2} \rho^{2}\right)
$$

Furthermore,

$$
F_{12}=R_{12}-\frac{1}{\ell^{2}} e_{[1} e_{2]}
$$

We will consider the theory in the limit that $b$ is held fixed while $g \rightarrow 0$ and $\ell \rightarrow \infty$. Clearly, then $F_{12} \rightarrow R_{12}$, the mass term in the quadratic operator vanishes and the action becomes

$$
S=\int d^{2} x \frac{1}{\sqrt{2}} \sqrt{g} R \rho+b^{2} \rho(-\bar{\square}) \rho+\frac{1}{4 g^{2}} R^{2}+\frac{1}{b^{2}}\left(D_{[1} e_{2]}\right)^{2}
$$

where we have substituted the relation $\int R_{12}=\int \frac{1}{2} \sqrt{g} R$. Notice that the term involving $R^{2}$ plays no role in the IR while the torsion term survives in this limit. Finally, we make the crucial observation that in two dimensions any metric is conformally equivalent to a flat metric. Furthermore, in this case, the Laplacian in the curved metric is simply related to the flat space Laplacian $\bar{\square}$ via the relation $\sqrt{g} \square=\bar{\square}$. This allow us to replace the flat Laplacian occuring in eqn. 4.1 by its curved 
space equivalent which then matches the linear term in $\rho$ which becomes $\sqrt{g} R$ and depends on the emergent curved metric.

We see that the action in unitary gauge resembles the Liouville action of two dimensional gravity with the radial mode $\rho$ replacing the conformal factor. Indeed it appears that we can now integrate out $\rho$ to produce the non-local form of the Polyakov action. However, the measure for the $\rho$ integration $\prod_{x} \rho(x)^{2} d \rho(x)$ is not translation invariant and so contrary to naive expectations the integral is not a pure gaussian. This is analogous to what happens in standard approaches to quantum Liouville theory where the measure for the conformal mode integration is also not translation invariant $[2,3]$. In that case transforming to a translation invariant measure one picks up a Jacobian which takes the form of the original action with renormalized coefficients. It is not clear how to follow the same procedure in this case.

However the original gauge invariant action written in terms of the field $\phi$ does not suffer from these problems and so our claim is that this lattice gauge theory at some suitable critical point should yield the same physics as the continuum quantum gravity model. How this is occur we will address in the next section.

\section{Tuning the coefficient $b$}

In the original gauge invariant lattice action I can simply integrate out the $\phi$ field to produce an effective action for the $S U(2)$ gauge field of the form

$$
S_{\mathrm{eff}}=\frac{1}{4 b^{2}} \sum_{i j} F_{12}(i)\left(-\bar{\square}^{-1}\right)_{i j} F_{12}(j)
$$

Furthermore, from our arguments in the previous section, we expect this to approach the Polyakov action in eqn. 1 as $\beta \rightarrow \infty$ and $L \rightarrow \infty$ with $b$ fixed. The remaining question is what determines the particular value of $b$ that corresponds to the correct conformally invariant theory of quantum gravity. Our answer is that it is determined by searching for a phase transition in the Yang-Mills system corresponding to the spontaneous breaking of the $Z_{2}$ center symmetry. The latter is defined by

$$
U_{\mu}(x) \rightarrow Z U_{\mu}(x)
$$

where $Z \in \pm 1$ is a $Z_{2}$ phase. The lattice action in invariant under this discrete, global symmetry but there remains the possibility that it is broken spontaneously as we vary $c$. Indeed, we observe this to be the case as can be seen in fig. 1 where we plot the distribution of the Polyakov line for several values of $b$ as determined by a Monte Carlo simulation. Notice that the Polyakov line is not invariant under the center transformation $U_{\mu}(x) \rightarrow Z U_{\mu}(x)$ with $Z \in \pm 1$ and hence serves as an order parameter for such a transition. Clearly for large $b$ the distribution is centered about the origin corresponding to an unbroken center symmetry. Conversely for small $b$ the distribution consists of two peaks at $P= \pm 1$ as expected for broken center symmetry. The transition between these two regimes can be estimated by finding a value of $b$ at which the distribution is close to uniform. This is seen for $1.1<b<1.3$ as shown in the figure. The critical value of $b_{c}=\sqrt{\frac{12 \pi}{25}}=1.228$ [12] is clearly consistent with this estimate of the critical coupling for a center breaking transition. 


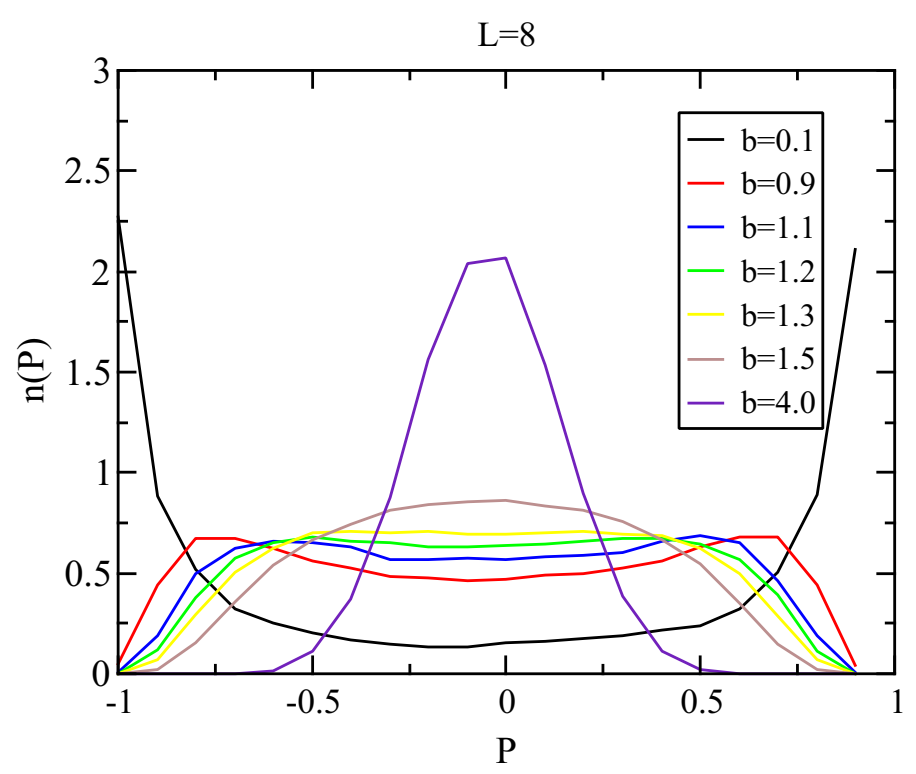

Figure 1: Distribution of the Polyakov line for several values of $b$ for an $8 \times 8$ lattice

\section{Conclusions}

In this paper we have shown how to generalize the gauge theoretic construction of gravity pioneered by MacDowell and Chamseddine and presented in [9] to include derivative terms in the scalar field and how such a generalization is capable of reproducing the Liouville/Polyakov theory of $2 \mathrm{~d}$ quantum gravity.

Furthermore we show how to do this in the context of a lattice gauge theory which avoids issues of gauge fixing and allows us to measure the critical value of the parameter arising in the gravity theory by tuning to a phase transition in the lattice model. It is quite remarkable that the lattice theory knows about the ghost contributions that arise after gauge fixing the diffeomorphisms in the usual metric approach to quantum gravity. In contrast to previous gauge theoretic approaches to gravity we do not seek to give a vev to the scalar field that appears in the formulation but instead force the vev to vanish as a result of strong IR fluctuations which arise as a result of the logarithmic interaction that arises in the effective theory. This strategy is very much in the same spirit as arise in the work of Mottola et al [12].

\section{Acknowledgments}

SMC acknowledges the support of the DOE through grants DE-SC0019139 and DE-SC0009998 and conversations with Jay Hubisz. 


\section{References}

[1] A. M. Polyakov, “Quantum Geometry of Bosonic Strings,” Phys. Lett. 103B, 207 (1981). doi:10.1016/0370-2693(81)90743-7

[2] F. David, "Conformal Field Theories Coupled to 2D Gravity in the Conformal Gauge," Mod. Phys. Lett. A 3, 1651 (1988). doi:10.1142/S0217732388001975

[3] E. D’Hoker and P. S. Kurzepa, “2-D Quantum Gravity and Liouville Theory,” Mod. Phys. Lett. A 5, 1411 (1990). doi:10.1142/S0217732390001608

[4] P. Di Francesco, P. H. Ginsparg and J. Zinn-Justin, “2-D Gravity and random matrices," Phys. Rept. 254, 1 (1995) doi:10.1016/0370-1573(94)00084-G [hep-th/9306153].

[5] J. Ambjorn, P. de Forcrand, F. Koukiou and D. Petritis, "Monte Carlo Simulations of Regularized Bosonic Strings,” Phys. Lett. B 197, 548 (1987). doi:10.1016/0370-2693(87)91053-7

[6] D. V. Boulatov, V. A. Kazakov, I. K. Kostov and A. A. Migdal, "Analytical and Numerical Study of the Model of Dynamically Triangulated Random Surfaces,” Nucl. Phys. B 275, 641 (1986). doi:10.1016/0550-3213(86)90578-X

[7] S. M. Catterall, J. B. Kogut and R. L. Renken, "Scaling behavior of the Ising model coupled to two-dimensional quantum gravity,” Phys. Rev. D 45, 2957 (1992). doi:10.1103/PhysRevD.45.2957

[8] S. W. MacDowell and F. Mansouri, "Unified Geometric Theory of Gravity and Supergravity,” Phys. Rev. Lett. 38, 739 (1977) Erratum: [Phys. Rev. Lett. 38, 1376 (1977)]. doi:10.1103/PhysRevLett.38.1376, 10.1103/PhysRevLett.38.739

[9] A. H. Chamseddine and D. Wyler, "Gauge Theory of Topological Gravity in (1+1)-Dimensions," Phys. Lett. B 228, 75 (1989). doi:10.1016/0370-2693(89)90528-5

[10] E. Witten, “(2+1)-Dimensional Gravity as an Exactly Soluble System,” Nucl. Phys. B 311, 46 (1988). doi:10.1016/0550-3213(88)90143-5

[11] P. O. Mazur and E. Mottola, "Weyl cohomology and the effective action for conformal anomalies," Phys. Rev. D 64, 104022 (2001) doi:10.1103/PhysRevD.64.104022 [hep-th/0106151].

[12] E. Mottola and R. Vaulin, "Macroscopic Effects of the Quantum Trace Anomaly,” Phys. Rev. D 74, 064004 (2006) doi:10.1103/PhysRevD.74.064004 [gr-qc/0604051].

[13] S. Catterall and E. Mottola, “The Conformal mode in 2-D simplicial gravity,” Phys. Lett. B 467, 29 (1999) doi:10.1016/S0370-2693(99)01053-9 [hep-lat/9906032]. 